zu einem Krystall etwas grünen Vitriol, dann Wasser und Schwefelsăure, so erhălt man die bekannte Reaction anf Salpetersăure.

Wir abergehen weitere Reactionsversuche, deren wir noch mehrere mittheilen konnten. Das Gesagte mag vor der Hand genugen zum Beweise, dals das Stibăthyl alle Eigenschaften eines selbstständigen Radicales, gleich dem Kakodyl, besitzt, und wir hoffen bald năhere Mittheilungen uber die verschiedenen Verbindungen machen zu konnen. Es unterliegt keinem $Z$ weifel, dafs auch Methyl und Amyl auf gleiche Weise mit dem Antimon verbunden werden könuen; auch lassen sich wabrscheinlich andere Mctalle nach derselben Methode in organische Verbindungen überfäbren.

\title{
1II. Ueber die Entladung der Franklin'schen
}

Batterie; pon P. Riefs.

Die nach Franklin's Vorschlag aufgestellte elektrische Battcrie besteht aus einzelnen isolirten leydener Flaschen (oder Batterien), die in eine Reihe gestellt und so mit einander verbunden sind, dafs der Knopf der ersten Flasrhe mit dem Conductor der Elektrisirmaschine, der Knopf jeder folgenden Flasche mit der äufsern Belegung der ihr in der Reihe vorangehenden, die äufsere Belegung der letzten Flasche mit der Erde in leitender Verbindung steht. Wird von dem Conductor positive Elek tricität dem Inneren der ersten Flasche zugeführt, so geht von ihrer äufseren Belegung positive Elektricităt fort und hăuft sich im Inneren der zweiten Fasche an, von deren hufseren Belegung positive Elektricität in die dritte Flasche geht und so fort. Nachdem in dieser Weise alle Flaschen geladen wurden, kann man dic Verbindungen derselben aufheben und, wie in der gewöhnlichen Batterie, alle innere Bele- 
gungen der Flaschen mit einander, und ebenso alle äufsere Belegungen verbinden, um sie sodann gemeinschaftlich 20 cntladen. Diese Batterie, auch Cascadenbatterie und Flaschensăule genannt, hat nicht den practischen Werth, den man ihr beigelegt hat. Obgleich nur die erste Flasche direct vom Conductor geladen wird, so erfordert diefs, wenn es auf stärkere Ladungen ankommt, mehr Zeit und eine viel gröfsere Wirksamkeit der Elektrisinnaschine, als wenn alle Flaschen direct zu demselben Grade geladen würden.

Franklin, dem nicht leicht Etwas entging, das in Kreise seiner Beobachtungen lag, hatte diefs wohl bemerkt und seine Batterie verworfen, weil die Flaschen einiges Widerstreben (some reluctance) äufserten, sich laden $\mathrm{z11}$ lassen '). Diefs ist von den Physikern nicht beachtet worden, die sich nach ihm mit der Batterie beschäfigt haben. Neuerdings hat die Batterie ein theoretisches Interesse erhalten, indem Dove ibre Entladung versuchte bei dersel. ben Anordnung, die zu ihrer Ladung dient, und in Bezug auf Schlagweite und Erwänung sehr einfache Gesetze aufstellte ${ }^{2}$ ). Ich habe, in Folge dieser Angabcn, Versuche angestellt, dic ich ausführlich mittheilen will, che ich die Gründe angebe, die mich abhielten, aus ihnen ein Gesetz abzuleiten ${ }^{3}$ ).

1) Experim. and observat. 5th ed. p. 25.29.

2) Poggend. Annalen 72. 406.

3) Hr. Knochenhauer hát vor Kurzem eine Minge hiehergehöriger Beobachlungen mitgetheilt und durch complicirte Formeln darzustellen rersucht. (Annalen 79. 354). Ich mufs diese Ablandlung auf sich berulhen lassen, wie ich es bereits mit der grofsen Zalal vorhergehender Arbeiten desselben Verfassers zu thun für nötlig fand. Diese Ablıandlungen enthalten nämolich sehr verwickelte Versuche, dic auf die willkürlichste Weise, häufig unter Vernarlhlässigung bekannter und bewïhrter Sätze der Elekıricitätslelare, zil Angriffen auf nenerdings gewonnçe Erfalırungen benutzt werden. Als Vertheiliger dieser Säıze und Eıfalırungen aufzutreten, wïtde ich nur dann fïr gerechtertigt halten, wenn mir die Angriffe an sich gefilırlich erschienen, oder wenn sie irgendwo Anklang finden. Dals Letaleres bisher nicht der Fall gewesen, ist aus den Repertorien der Pliysik, am klarsten aus Miller's Bericht über die neusten Fortschritte der Physik (Braunsrhweig 1849) zu crselien, in 


\section{Versuche im schliefrungsbogen.}

Vier leydener Flaschen, jede ron $1 \frac{1}{2} \square$ Fufs Belegung, wurden auf $4_{T}^{\prime} \frac{1}{2}$ Zoll breite, auf Glasfafsen isolirte, Melallteller gestellt und durch drei Kupferdrabte (von 21 Zoll Länge $T^{7}$ Lin. Dicke) zu einer Franklin'schen Batterie verbunden wie Fig. 5, Taf. IV. zeigt. Mit dem Knopfe $i_{1}$ war der Conductor der Maschine wăhrend der Ladung in Verbindung gesetzt, von der äufseren Belegung $a_{4}$ ging ein Draht zur Maafsflasche, deren Kugeln $\frac{1}{2}$ Linie von einander standen. Es wurden Ladungen der Batterie angewandt, die 1 bis 3 Entladungen der Maalsflasche verursachten. Bei Versuchen, die mit einander verglichen wurden, blieb die Ladung constant. Nach der Ladung wurde der Schliefsungsbogen angelegt, der, wo es nicht anders gesagt ist, vollkommen zur Erde abgeleitet war. Das eine Ende des Bogens wurde durch den Entladungsapparat mit dem Knopfe $i_{1}$ der ersten Flasche verbunden, nachdem das andere Ende an die auufsere Belegung des ersten, zweiten u. s. f. Flasche angelegt worden war, was durch die Angabe bezeichnet wird, dafs eine, zwei u. s. f. Flaschen benutzt werden. Vor der Eutladung der benutzten Flaschen wurden die übrigen von ihnen getreunt, oder, was merklich denselben $\mathbf{E r}$ folg hatte, ohue Lösung der Verbindung entladen.

Eroärmung. Versuch I. Ein empfindliches elcktrisches Thermometer wurde in den Schliefsungsbogen eingeschaltet. Die Batterie wurde geladen bis zwei Funken an der Maalsflasche übergegangen waren.

\begin{tabular}{crrrc} 
& \multicolumn{3}{c}{$\begin{array}{c}\text { Erwärmung in Schlic- } \\
\text { Bsungsbogen. }\end{array}$} & Verhältaifs d. Mittel. \\
Benutzte Flaschen. & \multicolumn{7}{c}{$\mathbf{7 , 5}$} & $\mathbf{7 , 4}$ & $\mathbf{7 , 7}$ & $\mathbf{1}$ \\
1 & 12,8 & 11,9 & 12,8 & 1,7 \\
2 & 15,4 & 15,3 & 15,8 & 2,0 \\
3 & 21,6 & 22,3 & 21,4 & 2,9
\end{tabular}

welchem letzern Werke auch der Werth der, einem Aücluigen Leser vielleicht auffallenden, Uebereinstinnmung von Kno ch e n h a uer's Formeln mit seinen Benbachtungen in das rechte Licht gestellt worden ist. 
Während also 1 bis 4 Flaschen benutzt wurden, nahm die Erwärmung von 1 bis nahe $3 \mathrm{zu}$.

Versuch II. Die absolute Grölse der Erwärmung hängt von der Beschaffenheit des Schliefsungsbogens ab. Als der Bogen durch einen Platindraht von 116 Lin. Lange, 0,0185 Halbmesser verlăngert war, wurden bei derselben Ladung, wie früher, die folgenden geringeren Erwärmungen betrachtet.

\begin{tabular}{|c|c|c|c|c|}
\hline nutzle F & \multicolumn{3}{|c|}{$\begin{array}{c}\text { Erwörnung im Schlie- } \\
\text { fsungsbogen. }\end{array}$} & Verbältnifs d. Mitt \\
\hline 1 & 4,6 & 5,0 & 5,0 & 1 \\
\hline 2 & 8,2 & 8,0 & 8,0 & 1,7 \\
\hline 3 & 11,8 & 11,4 & 11,2 & 2,3 \\
\hline 4 & 14,6 & 15,3 & 15,1 & $\mathbf{3 , 0}$ \\
\hline
\end{tabular}

Versuch III. Ebenso hăngt aber die Erwärmung in Schliefsungsbogen von der Beschaffenheit der Zwischendrähte $a b$, welche die einzelnen Flaschen mit einander verbinden. Der Platindraht wurde aus dem Schliefsungsbogen entlernt und zum ersten Zwischendrahte $\left(a_{1} i_{2}\right.$ der Figur 5 hinzugesetzt.

\begin{tabular}{|c|c|c|c|c|}
\hline Benutzte Flas & \multicolumn{3}{|c|}{$\begin{array}{c}\text { Erwärmung in Schlie- } \\
\text { Isungsbogen. }\end{array}$} & Verhälınifs d. Mittel. \\
\hline 1 & 7,5 & 7,3 & $\mathbf{7 , 5}$ & - \\
\hline 2 & 7,8 & 7,3 & 7,9 & 1,7 \\
\hline 3 & 11,0 & 11,0 & 11,0 & 2,4 \\
\hline 4 & 13,2 & 11,0 & 13,6 & $\mathbf{3 , 0}$ \\
\hline
\end{tabular}

Die erste Horizontalreihe gehört begreiflich nicht bierher, da bei Benutzung Einer Flasche kein Zwischendraht vorhanden ist.

Versuch IV. Es wurden drei Batterien, von denen jede nahe $3 \square$ Fufs innerer Belegung enthielt, einzeln isolirt und zu einer Franklin'schen Batterie mit einander verbunden. Man hat also in Fig. 5 die vierte Flasche entfernt und $a_{3}$ mit der Maafsflasche verbunden zu denken. Bei der Ladung gingen 3 Funken in die Maafsflasche über. 


\begin{tabular}{cccc} 
Benutzte Batterien. & \multicolumn{2}{c}{$\begin{array}{c}\text { Erwärmung im Schlie- } \\
\text { fsungshogen. }\end{array}$} & Verhältnifs d. Mittel. \\
1 & $\mathbf{7 , 3}$ & $\mathbf{7 , 3}$ & $\mathbf{1}$ \\
2 & $\mathbf{1 3 , 3}$ & $\mathbf{1 3 , 0}$ & $\mathbf{1 , 8}$ \\
3 & 17,7 & $\mathbf{1 8 , 5}$ & $\mathbf{2 , 5}$
\end{tabular}

Aus den Versuchen I bis IV. folgt, dafs die Erwärmung im Schliefsungsbogen der hier angewandten Batterie in geringerem Verbältnisse wăchst, als die Zabl der benutzten Elemente.

Schlagweite. Es wurde die Batterie aus $\&$ Flaschen (Vers. I.) angewendet, im Schliefsungsbogen aber an der Stelle des Thermometers ein Funkenmikrometer angebracht. Die Verbindung des Bogens mit den Flaschen wurde auf verschiedene Weise bewerkstelligt.

Versuch V. Die eine Kugel des Mikrometers wurde mit dem Knopf $i_{1}$ der ersten Flasche verbunden, die audere Kugel mit einer der ăufseren Belegungen. Die Kugeln wurden in solcher Entfernung von einander gestellt, dafs zwischen ihnen ein Funke überging zugleich mit dem Funken der Maafsflasche, der die bestimmte Ladung der Batterie auzeigte. Die Drähte, welche das Mikrometer mit den Flaschen verbanden, waren in der ersten Versuchsreihe so lang, dafs das Mikrometer bei allen Versuchen an derselben Stelle stehen bleiben konnte, in der zweiten wurde der eine Draht bis 3 Zoll verkurzt, so dafs das Mikrometer stets sehr nahe der aufsern Belegung der letzten benutzten Flasche stand. Die Schlagweiten sind nur bis 0,1 Linie gesucht, so dals also die in der folgenden Tabelle angegebenen Weiten, um 0,1 vermehrt, keine Entladung im Mikrometer zuliefsen.

Benutzte Flaschen. Sehlagweite in par. Lin. Verhälınifs d. Mittel.

$\begin{array}{llll}1 & 0,4 & 0,4 & 1 \\ 2 & 1,0 & 1,0 & 2,5 \\ 3 & 1,7 & 1,7 & 4,2 \\ 4 & 2,5 & 2,6 & 6,4\end{array}$

Diese Untersuchurgsart giebt zu grofse Schlagweiten, weil dabei der Schliefsungsbogen nothwendig isolirt ist, und

Poggendorf's Annal. Bd. LXXX. 
kurz vor dem Ueberspringen des Funkens in der Maafsflasche im Innern der ersten Battericflasche einc gröfsere Dichtigkeit berrscht, als der daselbst befindlichen Elektricitätsmenge zukommt. Es wurde daher die folgende bessere Methode zur Bestimmung der Schlagweite angewandt.

Versuch VI. Die eine Kugel des Mikrometers wurde mit dem Entladungsapparat, die andere mit einem zur Erde abgeleiteten Drahte verbunden. Nachdem die Batterie die ihr bestimmte Ladung erhalten batte, wurde der abgeleitete Drabt an eine der äufseren Belegungen der Batterie angelegt und, nach Entladung der ausgeschlossenen Flaschen, durch den Entladungsapparat die Verbindung der isolirten Mikrometerkugel mit dem Knopfe $i_{\downarrow}$ der ersten Flasche hergestellt. Es wurde die gröfste Entfernung der Kugeln gesucht (bis 0,1 Lin.) bei welcher eine Entladung durch das Mikrometer stattfand.

$\begin{array}{ccc}\text { Benutzte Flaschen. } & \text { Sclılagweite in Linien. } & \text { Verhïlınis. } \\ 1 & 0,3 & 1 \\ 2 & 0,9 & 3 \\ 3 & 1,4 & 4,7 \\ 4 & 2,3 & 7,7\end{array}$

Versuch VII. Am leichtesten wurde die Schlagweite gefunden, wenn die eine Mikrometerkugel mit einem isolirten drehbaren Drahte, die andere mit einem zur Erde abgeleiteten Drahte verbunden war. Nach der Ladung der Batterie wurde der erste Draht uit dem Knopfe der ersten Flasche, der zweite mit einer äufseren Belegung in Berührung gesetzt, und durch behutsames Nähern der Kugeln des Mikrometers eiue Entladung der Batterie herbeigeführt.

$\begin{array}{cccc}\text { Benutzle Flaschen. } & \text { Sclılagweite in Linien. } & \text { Verhälınifs d. Mittel. } \\ \mathbf{1} & \mathbf{0 , 3} & \mathbf{0 , 3} & \mathbf{1} \\ \mathbf{2} & \mathbf{0 , 8 5} & \mathbf{0 , 9 5} & \mathbf{3} \\ 3 & \mathbf{1 , 4} & \mathbf{1 , 4} & \mathbf{4 , 7} \\ 4 & \mathbf{2 , 2} & \mathbf{2 , 3} & \mathbf{7 , 5}\end{array}$

Versuch VIII. An der in Versuch IV gebrauchten, aus 
3 Elementen bestehenden, Batterie wurden folgende Schlagweiten gefunden.

$\begin{array}{ccc}\text { Benutzte Elemente. } & \text { Schlagweite. } & \text { Verbältmifs. } \\ 1 & 0,4 & 1 \\ 2 & 1,2 & 3 \\ 3 & 1,8 & 4,5\end{array}$

In den Versuchen $\mathrm{V}$ bis VIII ist bei Benutzung von 1 bis 3 Elementen die Schlagweite noch nicht bis zum fänfachen, bei Benulzung von 1 bis 4 Elementen nicht bis zum achtfachen gestiegen.

Versuche im $\mathbf{Z}$ wischendrahte.

Versuch IX. In der aus 4 Flaschen bestehenden Batterie (Fig. 5.) wurde das Thermometer in den Zwischendraht $a_{1} i_{2}$, zwischen der ersten und zweiten Flasche, eingeschaltet und die Erwărmung bei der Entladung der Batterie durch den Scbliefsungsbogen beobachtet. Es konnte hier nur die Ladung gebraucht werden, die 1 Funken in der Maafsflasche erzeugte, weil bei stärkerer Ladung Elektricităt am Thermometer ausströmte. Bei Anwendung Einer Flasche wurde der Schliefsungsbogen binter den Thermometer angelegt, so dafs sich letzteres im Schliefsungsbogen befand.

$\begin{array}{lcccc}\text { Benutzte Flaschen } & 1 & 2 & 3 & 4 \\ \text { Erwärmung } & 3 & 4 & 5,5 & 7,5 .\end{array}$

Versuch X. An die Stelle des Thermometers wurde das Funkenmikrometer gesetzt, so dafs also der Zwischendraht unterbrochen war; während der Ladung wurde die Lücke durch ein Drahtstück ausgefüllt, das darnach isolirt entfernt wurde. Es wurde die grofste Entfernung der Kugeln des Mikrometers gesucht, bei welcher die Entladung der Batterie durch den Schliefsungsbogen einen Funken im Mikrometer erzeugte. Diese Entfernung giebt die Schlagweite in Zwischendrahte. 


$\begin{array}{ccc}\text { Benutzle Flaschen. } & \text { Schlagwreite in Linien. } & \text { Verhälınils. } \\ 1 & 0,3 & 1 \\ 2 & 0,9 & 3 \\ 3 & 1,4 & 4,7 \\ 4 & 2,2 & 7,3\end{array}$

Versuch XI. Um genauere Werthe der Erwärmung zu erhalten wurde die Batterie aus 3 Elementen (Versuch IV) gebraucht, der eine Ladung gegeben wurde, bei welcher in der Maafsflasche 3 Funken übergingen.

Benutale Elemente.

1

2
Erwärmung.

88

$13,5 \quad 13,6$

1920
Verhältenifs d. Mituel.

1

1,7

2,4

Versuch XII. Im ersten Zwischendrahte dieser Batterie wurden die folgenden Schlagweiten gefunden. Die Ladung war in der zweiten Reibe grölser als in der ersten.

Benutzte Elemente. Schlagweite in Linien. Verhältnifs d. Mittel.

$\begin{array}{ccccc}1 & 0,25 & 0,4 & & 1 \\ 2 & 0,80 & 1,2 & & 3,1 \\ 3 & 1,10 & 1,7 & & 4,3 .\end{array}$

Diese Versuche im Zwischendrahte entsprechen genau den im Schliefsungsbogen angestellten. Auch hier nimmt die Erwärmung in geringerem Verhältnisse zu, als die Zahl der benutzten Elemente, und die Schlagweite erreicht bei 3 Elementen nicht das fänffache, bei 4 Elementen nicht das achtfache der Schlagweite, die bei Benuli:ng eines Elementes gefunden wurde.

Versuche an einer Batterie mit ungleichen Elementen.

Bisher war die Batterie aus gleichen Elementen zusammengesetzt; jedes Element bestand aus einer Flasche, oder aus mehreren gleichartig mit einander verbundenen Flaschen. Nun wurde eine Batterie aus 2 Elementen gebildet und die Grölse des einen Elementes verändert.

Versuch XIII. Zum ersten (an dem Couductur der Maschine anliegenden) Elemente wurde eine Flasche von $1 \frac{1}{2}$ $\square$ Fufs Belegung genommen und das zweite Element aus 
1 bis 4 gleichen Flaschen zusammengesetzt (Fig. 6.) Der Zwischendraht $a_{1} i_{2}$ war hier, wie früher, 21 Zoll lang, iz Lin. dick. Es wurden folgende Erwärmungen an einem im Schliefsungsbogen eingeschalteten Thermometer bei gleicher Ladung der Batterie beobachtet.

Zahl der Flaschen des zweiten Elementes $\boldsymbol{s}_{2}$

1

2

3

4
Erwärmung

A.

$23,8 \quad 24$

$19,0 \quad 18,3$

$17,3 \quad 17,0$

$16,2 \quad 16,5$
${ }_{\theta} \sqrt{8_{2}}$.

23,9

26,3

29,6

33,6 .

Versuch XIV. Das zweite Element wurde constant genommen, das erste hingegen aus 1 bis 4 Flaschen gebildet. (Fig. 7.) Am Schliefsungsbogen wurden folgende Erwärmungen beobachtet.

\begin{tabular}{clll}
$\begin{array}{c}\text { Zahl der Flaschen des } \\
\text { ersten Elementes } \text { s. }_{1}\end{array}$ & \multicolumn{2}{c}{ Erwärnuug } & \multirow{2}{*}{$\boldsymbol{\theta} \sqrt{\mathbf{s}_{1}}$} \\
1 & $\mathbf{2 4}$ & & $\mathbf{2 4}$ \\
2 & 18,5 & 18,2 & $\mathbf{2 5 , 9}$ \\
3 & 16,4 & 16,6 & 28,6 \\
4 & 15,8 & 15,7 & $31,4$.
\end{tabular}

In beideu Versuchen nimmt die Erwärmung mit vermehrter Flaschenzahl des veränderlichen Elementes ab, so aber, dals das Product der Erwărmung in die Quadratwurzel der Zahl der Flaschen merklich zunimint.

Die Resultate der mitgetheilten Versuche weichen bedeutend von den Gesetzen ab, die Dove in der oben angeführten Abhandlung als allgemeingültig für die Franklin'sche Batterie aufgestellt bat.

Die Erwärmung sowohl im Schliefsungsbogen als im Zwischendrabte soll proportional der Zahl der gleichen Elemente zunehmen, die zur Entladung benutzt werden; hier ist die Erwărmung in geringerem Verhältnisse wachsend beobachtet worden. Bei 1 bis 4 Elementen stieg die Erwärnung nur von 1 bis 3 . (Vers. I bis IV, IX, XI.) 
Die Schlagweiten im Schliefsungsbogen und Zwischendraht sollen im Verbältnisse der Quadrate der benutzten Elemente stehen, hier ist diefs Verbaltnils viel kleiner gefunden worden. Bei 1 bis 4 Elementen nahm die Schlagweite von 1 bis hochstens 7,7 zu (Vers. V. bis VIII, X, XII.)

Bei verănderter Grölse eines Elements einer aus zwei Elementen bestehenden Batterie soll das Product der Erwärmung im Schliefsungsbogen in die Quadratwurzel der Flächengrofrse des Elementes constant seyn; hier ist das Product mit steigender Flächengröfse zunehmend gefunden worden (Vers. XIII, XIV.) Diese Abweichungen werden nicht auffallen, wie die folgende Auseinandersetzung gezeigt haben wird, dafs eine unbestimmte Aufgabe vorgelegen hat, für die also keine allgemeine Lösung zu erwarten ist.

Orsache der Entladungserscheinunged der Franklin'schen Batterie.

Eine leydener Flasche nimmt in ihrem Inneren so lange Elektricităt auf, bis ibr Knopf eine bestimmte elektrische Dichtigkeit erreicht hat. Die aufgenominene Elektricitätsmenge ist sehr geriug, wenn die äufsere Belegung der Flasche isolirt ist, wird um desto gröfser, ein je längerer Draht an die äufsere Belegung angelegt worden, und erreicht ihr Maximum, wenn der Draht mit der Erde in Verbindung gesetzt worden ist. Es folgt hieraus, was sich auch leicht direct nachweisen liefse, dafs bei einer bestimmten Elektricitätsmenge im Inneren der Flasche die Dichtigkeit an ihrem Knopfe desto gröfser ist, eine je geringere Ableitung ihre äufsere Belegung erbalten bat. In der That ist nun die Franklin'sche Batterie, in der Art benutzt, wie es die aufgefübrten Versuche zeigen, nichts anderes als eine solche einzelne Flasche mit veränderliches Ableitung ihrer äufseren Belegung. Aus je mehr Elementen eine solche Batterie bestebt, desto 'geriuger ist die Ableitung, desto mehr Widerstreben, nach Frankliu's Ausdruck, bat ihr erstes Element gegen die Ladung, und desto gröfser ist bei constanter Ladung die elektrische Dichtigkeit an Knopfe dieses Ele- 
ments. Hat man daher eine Batterie aus 4 Elementen (Fig. 5.) geladen, und benutzt nur 3 Elemente bei der Entladung, iudem man den Schliefsungsbogen an $a_{3}$ anlegt, so sinkt augenblicklich die Dichtigkeit am Knopfe $i$, und man mufs daher eine kleinere Schlagweite finden, als wenn der Bogen an $a_{4}$ angelegt worden wäre. Aus gleichem Grunde mufs die Schlagweite noch kleiner seyn, wenn der Bogen an $a_{3}$ und endlich an $a_{1}$ angelegt wird. Man könnte glauben, dafs ein allgemein gültiges Gesetz für diese Abnahme der Schlagweite zu finden wäre, wenn die Bedingung gestellt ist, dafs alle Elemente der Batterie gleichartig und gleichgrofs, alle Isolirteller und $\mathrm{Zwischendrähte} \mathrm{gleich} \mathrm{ge-}$ nommen werden. Diefs ist nicht der Fall. Ich habe in einer früheren Untersuchung ') einen ähnlichen aber viel einfachereren Fall behandelt. Eiuer elektrisirten Metallscheibe war eine gleiche, vollkommen abgeleitete Scheibe parallel gegenübergestellt, und ich suchte die Schlagweite oder die ihr proportionale elektrische Dichtigkeit an einem Punkte der elektrisirten Scheibe bei verschiedener Entfernung der Scheibe von einander. Die Abnahme der Dichtigkeit mit zunehmender Entfernung zeigte sich verschieden nach der absoluten Gröfse der Scheiben, nach der Lage des Punktes, an dem die Dichtigkeit untersucht wurde, und endlich nach der Lage des Ableitungsdrahtes der zweiten Scheibe, so dafs ein Gesetz für diese Abnahme nicht aufgestellt werden konnte. Es ist daraus zu schliefsen, dafs bei der Frauklin'schen Battere die Abnahme der Schlagweite des ersten Elementes nach der Zahl der benutzten Elemente abbängig seyn wird von der Gestalt und absoluten Grölse der Elemente und Isolirteller und von der Form und Läuge der Zwischendräbte, so dafs ein Gesetz dieser Abuahme nur für dell angewaudten Apparat Geltung hat. Dasselbe, was für die Dichtigkeit am Knopfe des ersten Elementes, also für die Schlagweite im Schliefsungsbogen, gilt auch für die Dichtigkeit am Knopfe eines fol-

1) Pä ь. Inn. Bd. 73, 5. 379-398. 
genden Elementes oder die Schlagweite in einem Zwiscbendrahte der Batteric.

Die Erwărwung im Schliefsungsdrahte einer gewöhnlichen Batterie hăngt von der Elektricitătsmenge in der Batterie und der Schlagweite am Fortsatze der Batterie ab, an dem die Entladung vollführt wird, der Schlagweite sowohl, die der mittleren elektrischen Dichtigkeit der Batterie proportional, als der, die von der besonderen elektrischen Anordnung auf dem Fortsatze abhăngig ist, und die ich partikulăre Schlagweite nennen will. In Bezug auf die letztere habe ich gezeigt '), dafs dieselbe Batterie, durch Kugeln entladen, eine kleinere Schlagweite hat, als wenn der Entladungsfunke zwischen Scheiben übergeht, und dafs die Erwărmung im Schliefsungsbogen in zweiten Falle grölser ist, als im ersten. Dafs mit der Dichtigkeit in der Batterie, der die Schlagweite proportional ist, die Entladungsdauer der Batterie im umgekehrten Verhältuils stehe, habe ich zuerst vor dreizehn Jahren angenommen ${ }^{2}$ ) und seitdem durch eine grofse Menge von Versuchen bestäligt gefunden. Diese Versuche, aus welchen ich die Gesetze der elektrischen Erwärmung ableitete, bin ich auf das Sorgfältigste bemüht gewesen von einer Aenderung der partikulären Schlagweite frei zu balten. Deshalb wurden tuberall die Flächen, zwischen welchen die Entladung eintritt, ungeändert gelassen, und die Näherung dieser Flächen wurde niemals mit der Hand, sondern durch einen fallenden Körper ausgeführt. Ganz im Gegensatze hierzu ist es nun bei der Franklin'schen Batterie die partikuläre Schlagweite allein, die geändert wird, da man die mittlere Dichtigkeit der direct am Conductor geladenen Flasche bei zu vergleichenden Versuchen constant crbält. Die Schlagweite dieser Flasche wird nach der obigen Betrachtung geändert durch Aenderung der Ableitung ihrer äufseren Belegung, und ist unzweifelhaft die Ursache der Verschiedeuheit der beobachteten Erwärmungen. Es folgt hieraus, dafs cbenso we-

1) Pogg. Anu. 43. 79.

2) Pogg. Anu. 40. 351 . 
nig wie für die Schlagweite, ein allgemeines Gesetz der Erwărmuug nach der Zahl der benutzten Elemente der Batterie gefunden werden kann. Doch war es denkbar, dals unter gegebenen Bedingungen zwischen Scblagweite und Erwärmung eine bestimmte Beziehung stattfande, und so habe ich fruber beilaufig ') diese Beziehung angedeutet für den Fall, wo beide Erscheinungen in dem Zwischendrahte einer Franklin'schen Batterie beobachtet wurden. Auch diese Beziehung findet nach den hier vorgelegten Versuchen nicht allgemein statt und es ist darnach zu schliefsen, dafs Voraussetzungen, die in Bezug auf die mittlere elektrische Dichtigkeit einer geladenen Flasche gelten, nicht auf die Dichtigkeit einzelner Theile ihrer Belegung übertragen werden dürfen.

Für die Erwärmung und Schlagweite im Schliefsungsbogeu und Zwischendrahte einer aus gleichen Elementen bestehenden Franklin'schen Batterie lassen sich also keine Gesetze aufstellen, die nur die Zahl der benutzten Elemente in sich begreifen. Ein Gleiches gilt für eine aus $2 w c i$ Elementen bestehende Batterie, in welcher die Gröfse eines Elementes verändert wird, wie ein Blick auf Fig. 6 und 7 lehrt. In Fig. 6 ist es eine constante Flasche, deren äufsere Belegung eine Ableitung von 1 bis 4 Flaschen erhält, an deren Knopfe daher eine veränderliche partikuläre Schlagweite eintritt, welche die Ursache der im Scbliefsungsbogen beobachteten Erwärnungen ist. Zusammengeselzter ist der Fall der Fig. 7, der nur zum Theil bierher gehört. Es wird ein Element, das aus 1 bis 4 Flaschen besteht, am Conductor mit coustanter Elektricitätsmenge geladen; die mittlere elektrische Dichtigkeit des Elementes nimmt ab im Verhältnifs der zunebmenden Flaschenzabl und es müste daher nach dem allgemeinen Gesetze die Schlagweite und Erwärmung im gleichen Verhältnisse abnehmen. Nun aber bildet eine constante Flasche die Ableitung der äufseren Belegung dieses Elementes. Die Ableitung ist veränderlich im entgegengesetzten Sinu mit der Flaschenzahl des Ele1) $10 \mathrm{ggg} . \mathrm{Amn} .76 .484$. 
mentes denn offenbar ist Eine Flasche für Eine Flasche eine gröfsere Ableitung, als für 2, 3 oder 4 gleiche Flaschen. Da nun nach dem Obigen die partikuläre Schlagweite eines Elementes zunimmt mit Verminderung seiner Ableitung, so mufs Schlagweite und Erwärmung hier in einem kleineren Verhältnisse abnehmen, als dem der zunehmenden Flaschenzahl des Elementes. Ein allgemeines Gesetz dieser Abnahme kann hier, wie überall, wo partikuläre Schlagweiten im Spiele sind, nicht erwartet werden.

Die Betrachtung der Eutladung der Franklin'schen Batterie hat bisher den Einflufs der partikulären Schlagweite auf die Erwärmung gezeigt, einen Einflufs, der schon früher bekannt war und der sich einfacher und schlagender, als an der Franklin'schen, an der gewöhnlichen Batterie nachweisen läfst, indem man die Flächen äudert zwischen welchen der Entladungsfunke erscheint, oder die Wirkungen vou zwei gleichen Flaschen vergleicht, die cine vorschiedene Glasdicke besitzen. Aber ein Versuch ist noch nicht besprochen, der, wie die früheren, von Dove herrübrt, und eine merkwürdige Bestätigung ciues für die Entladung der Batterie überhaupt sehr wichtigen Satzes liefert. Wir habell gesehen (Versuch III.), dafs ein dem ersten Zwischendraht hinzugefïgler Platindraht die Erwärmung im Schliefsungsbogen sehr bedeutend verminderte. Da weder die Menge noch Dichtigkeit der Elektricität in Bogen verändert werden kann durch die Zeit, in welcher die Elektricitäten sich im Zwischendrahte ausgleichen, so beweist die beobachtete Verminderung der Erwärmung die Abbängigkeit, in welcher die auf einander folgenden Partialentladungen, welche dic Gesammtentladung bilden, von einauder stehen. Jedc Partialentladung einer Batterie kann erst dann eintreten, wemn die ilhr vorangehende Partialentladung vollendet, also die Elektricitiit in Schliefsungsbogen zur Ruhe gekommen und der anfingliche Ladungszustand der Batterie wiederhergestellt ist. Diefs ist der Satz, den ich aufgestellt ') und durch neuere Versuche uber die

1) Pogg. Ann. 78. 433 
Entladung in einem dauernd unterbrochenen Schliefsungsbogen bestătigt habe. Die Wirkung der Entladung der Franklin'schen Batterie ist dewnach folgendermafsen abzuleiten:

Die Wirkung im Schliefsungsbogen wird allein durch die Entladung des Elementes der Batterie hervorgebracht, an dessen innere Belegung der Schliefsungsbogen angelegt ist. Elektricitätsmenge, mittlere Dichtigkeit, partikuläre Schlagweite dieses Elementes haben denselben Einflufs auf die Entladung, wie in der gewöhnlichen Batterie. Jede Partialentladung führt eiue bestimmte Menge von Elektricität aus dem Inneren des Elementes in den Schliefsungsbogen; die Partialentladung ist vollendet und eine neue erfolgt, wenn jene Elektricitätsmenge den Schliefsungsbogen durchlaufen hat, und der Ladungszustand des Elements wiederhergestellt ist. Das Letztere geschiebt durch Fortführung einer dem Ladungszustand entsprechenden Elektricitätsmenge aus der äufseren Belegung des Elements, und wird in ersten Zwischendrahte zugleich mit der Ausgleichung der Elektricităten in den übrigen $Z$ wischendrähten ausgefübrt. Die Dauer jeder Partialentladung und damit die ihrer Summe, welche die Wirkung der Gesammtentladung bestiunt, ist daher abhängig von der Beschaffenheit sowohl des Schliefsungsbogens als der Zwischendrähte. Dasselbe gilt für eine Wirkung im Zwischendrahte, so dafs auch bier die Zeit, in welcher die cinzelnen Partialentladungen einander folgen, von der Zeit abhängt, in der die Ausgleichung der Elekricitäten in allen $Z$ wischendräbten stattgefunden, und die Entladung den ganzen Scbliefsungsbogen durchlaufen hat. 